history of preterm delivery. Multiple logistic regression and generalised additive models were used to explore the effect of covariates including area deprivation, smoking status, BMI, parity and ethnicity. Results The proportion of PTB was significantly different in the three groups: $1.35 \%$ (95\% CI 1.24 to $1.47, \mathrm{n}=38994)$ in the low risk group, compared to $6.55 \%$ (CI 6.09 to $7.03, n=10760$ ) in the medical disorder group and $9.2 \%$ (CI 7.39 to $11.61, \mathrm{n}=732$ ) in the previous preterm group. $64 \%$ of the women delivering at LWH were in the most deprived quintile relative to the English population. The unadjusted odds of preterm delivery in the most deprived quintile compared to the least was 1.60 (CI 1.28 to 2.00) in the uncomplicated group. In a multiple regression model, ever having smoked (OR 1.68 CI 1.35 to 2.08), underweight (OR $1.65 \mathrm{CI} 1.005$ to 2.56) and highest quintile of area deprivation (OR 1.59 CI 1.19 to 2.11) were associated with increased the risk of PTB. Being overweight decreased the risk of PTB (OR 0.76 CI 0.59 to 0.97). In the medical disorders group, age (OR $1.02 \mathrm{CI} 1.011$ to 1.04), highest quintile of area deprivation (OR $1.46 \mathrm{CI} 1.14$ to 1.88 ), underweight (OR $1.68 \mathrm{CI}$ 1.09 to 2.51), ever having smoked (OR $1.19 \mathrm{CI} 1.00$ to 1.44), nulliparity (OR $1.37 \mathrm{CI} 1.13$ to 1.66 ) and black ethnic group (OR $1.61 \mathrm{CI}$ 1.00 to 2.48) were associated with PTB.

Conclusions Preterm delivery contributes to inequalities in infant mortality. In a cohort of women with no identifiable risk factors for PTB at booking, deprivation of area of residence is associated with higher risk of PTB, even with adjustment for smoking and underweight, which are also important independent risk factors. Deprivation of area of residence needs to be considered when comparing obstetric outcomes in units around the UK.

\section{P17 BIRTH SIZE DIFFERENCES BETWEEN WHITE AND PAKISTANI ORIGIN INFANTS BY GENERATION: RESULTS FROM THE BORN IN BRADFORD COHORT STUDY}

doi:10.1136/jech.2010.120477.17

${ }^{1} \mathrm{~J}$ West, ${ }^{2} \mathrm{D}$ A Lawlor, 'L Fairley, ${ }^{1} \mathrm{~J}$ Wright, ${ }^{3}$ for the Born in Bradford Research Group. ${ }^{1}$ Bradford Institute for Health Research, Bradford Hospitals NHS Trust, Bradford, UK; ${ }^{2}$ MRC Centre for Causal Analyses in Translational Epidemiology, University of Bristol, Bristol, UK; ${ }^{3}$ Born in Bradford Project, Bradford Institute for Health Research, Bradford Royal Infirmary, Bradford, UK

Background Previous studies have shown marked differences in birth weight between babies born in the UK of South Asian origin and those of UK origin. Whether such differences persist across generations in contemporary populations, the mechanisms underlying them and the extent to which other dimensions of birth size vary between these two groups is unclear.

Objective To describe differences in term birth weight, head, arm and abdominal circumference and skinfolds between Pakistani origin and white British origin infants and to investigate whether the magnitude of any differences reduces depending on whether the parents and grandparents of Pakistani infants are born in the UK or Pakistan.

Design Birth cohort study (born in Bradford (BiB)).

Setting Bradford, UK.

Participants 1838 white British and 2222 Pakistani mothers recruited to $\mathrm{BiB}$ who completed a questionnaire at 26 weeks gestation and their babies born between September 2007 and November 2009.

Main Outcome Measures Birth weight, head, arm and abdominal circumference and skinfolds.

Results Pakistani babies were lighter (mean difference $227.6 \mathrm{~g}, 95 \%$ CI 198.3 to 256.8), had smaller head, arm and abdominal circumferences (mean differences $0.43 \mathrm{~cm}, 95 \%$ CI 0.30 to $0.56 ; 0.22 \mathrm{~cm}$, $95 \%$ CI 0.10 to $0.34 ; 1.25 \mathrm{~cm}, 95 \%$ CI 1.02 to 1.39 , respectively) and smaller subscapular and triceps skinfold thickness (mean differences
$0.22 \mathrm{~mm}, 95 \%$ CI 0.12 to 0.32 and $0.21 \mathrm{~mm}, 95 \%$ CI 0.13 to 0.29 ) than white British infants. Differences remained significant following adjustment for deprivation. Mean birth weight was highest in Pakistani infants when both parents were born in Pakistan (3206 g) and was lowest when both parents were UK born (3165 g).

Conclusions These results reaffirm that significant differences in birth size exist between white British and Pakistani origin infants in the UK. Despite the assumption that differences will reduce over successive generations, mean birth weight has not increased in infants of UK born Pakistani origin parents compared with infants of Pakistani born parents. This suggests that differences may be genetically determined or are affected by epigenetic or persisting behaviour characteristics. Further analysis will include adjustment for additional socioeconomic variables, other maternal and family characteristics and birthplace of maternal and paternal grandparents.

\section{P18 CHILD MALTREATMENT CO-OCCURRENCE AND ASSOCIATIONS WITH HOUSEHOLD DYSFUNCTION: EVIDENCE FROM THE 1958 BRITISH BIRTH COHORT}

doi:10.1136/jech.2010.120477.18

${ }^{1} \mathrm{R}$ Denholm, ${ }^{1} \mathrm{C}$ Power, ${ }^{2} \mathrm{C}$ Thomas, ${ }^{1} \mathrm{~L}$ Li. ${ }^{1} \mathrm{M}$ RC Centre of Epidemiology for Child Health/Centre for Paediatric Epidemiology and Biostatistics, UCL Institute of Child Health, London, UK; ${ }^{2}$ Division of Community Health Sciences, St George's University of London, London, UK

Background Child maltreatment has been associated with adverse health outcomes, including risk of mental health problems and cardiovascular disease. Little is known about how different forms of maltreatment co-occur and whether different patterns are associated with household dysfunction. Delineation of co-occurrence is important to establish in order that long-term health outcomes can be better identified and understood.

Objective To investigate (1) to what extent specific maltreatment subtypes co-occur in a British birth cohort and (2) how these patterns were associated with household dysfunction.

Design Longitudinal survey; the 1958 British birth cohort.

Setting England, Scotland and Wales.

Participants Individuals born during 1 week in March 1958. At age $45 \mathrm{y}, 78 \%$ of the remaining cohort (11971) completed questions on childhood experiences.

Outcomes Child maltreatment before age 16, including psychological, physical and sexual abuse and witnessing intimate partner violence, collected at age $45 \mathrm{y}$. Eleven indicators of parental neglectful behaviour, collected at 7,11, 16 and $45 \mathrm{y}$, were aggregated to derive a cumulative neglect score. Information on household dysfunction (eg, parental mental health, alcohol/drug misuse, poverty) was collected during childhood and at $45 \mathrm{y}$. OR presented were adjusted for sex and social class at birth.

Results Psychological abuse $(10.0 \%)$ was the most commonly reported maltreatment, followed by physical abuse (6.1\%), witnessing abuse (6.0\%) and sexual abuse (1.6\%); $24 \%$ had a neglect score $\geq 3$. $14 \%$ of participants experienced any one subtype of abuse. Of these, almost two thirds (64\%) experienced further abuse subtypes and/or had a neglect score $\geq 3$. Witnessing or experiencing abuse increased odds of reporting another maltreatment, for example, psychological and physical abuse OR 37.9 (95\% CI 30.8 to 46.5). The odds of reporting any abuse increased with neglect score; for example, for sexual abuse OR ranged from 1.5 (0.9 to 2.6) to 4.5 (2.5 to 8.1). Common household dysfunction variables (eg, conflict and physical punishment) were strong predictors for all abuse subtypes. Other dysfunction measures most strongly associated with maltreatment differed, for example, odds of psychological abuse were increased in association with mother/father with 
nervous trouble (OR 4.7 (4.1 to 5.4), OR 4.1 (3.4 to 4.8), respectively); odds of neglect were increased for those in institutional care (OR 3.8 (2.7 to 5.4)) or with a family member in prison (OR 3.8 (3.0 to 4.8$)$.

Conclusion Forms of maltreatment co-occurred in the cohort, such that cumulative exposure to neglect increased odds of abuse. Maltreated children had elevated exposure to household dysfunction.

\section{P19 SOCIOECONOMIC DISPARITIES IN TRAJECTORIES OF ADIPOSITY ACROSS CHILDHOOD}

doi:10.1136/jech.2010.120477.19

1,2 L D Howe, ${ }^{1} \mathrm{~K}$ Tilling, ${ }^{1} \mathrm{~B}$ Galobardes, ${ }^{1,2} \mathrm{G}$ Davey Smith, ${ }^{3} \mathrm{~A}$ R Ness, ${ }^{1,2} \mathrm{D}$ A Lawlor. ${ }^{1}$ Department of Social Medicine, University of Bristol, Bristol, UK; ${ }^{2}$ MRC Centre for Causal Analyses in Translational Epidemiology, University of Bristol, Bristol, UK; ${ }^{3}$ Department of Oral and Dental Science, University of Bristol, Bristol, UK

Objective Socioeconomic inequalities in obesity are consistently observed in high-income countries, with lower socioeconomic groups tending to be more adipose amongst both adults and children. The development of such inequalities across childhood, however, has not been studied using longitudinal data.

Design Using longitudinal data from a prospective birth cohort study, we modelled individual trajectories of adiposity from birth to 10 years using random effects linear spline models, and estimated differences in trajectories by socioeconomic position (measured by maternal education). Trajectories were modelled separately for boys and girls.

Setting Avon Longitudinal Study of Parents and Children, South West England.

Participants Participants were born in 1991/1992.

Main Outcome Measure Ponderal index (PI, $\mathrm{kg} / \mathrm{m}^{3}, \mathrm{~N}=12246$ ) from birth to 2 years and body mass index (BMI, $\left.\mathrm{kg} / \mathrm{m}^{2}, \mathrm{~N}=11380\right)$ from 2 to 10 years. Height and weight measures were from research clinics, health records, and parent-completed questionnaires; trajectory models included an indicator of measurement source to account for reduced accuracy of parent-reported measurements.

Results There was little socioeconomic patterning of PI from birth to 2 years. Socioeconomic differences in BMI began to emerge by 4 years old, and widened with increasing age. Amongst girls there was a clear gradient across all categories of maternal education by age 8 , with daughters of more educated women being less adipose. Amongst boys, sons of degree educated women had lower BMI, but there was little difference between the three lower maternal education categories. By 10 years old the mean BMI difference between the highest and lowest education category was $0.38 \mathrm{~kg} / \mathrm{m}^{2}$ for boys and $0.89 \mathrm{~kg} / \mathrm{m}^{2}$ for girls. The results imply that interventions to prevent inequalities in childhood obesity should begin in pre-school years.

\section{P20 DESCRIPTION OF DIETARY INTAKES OF ACRYLAMIDE IN THE BORN IN BRADFORD BIRTH COHORT STUDY}

doi:10.1136/jech.2010.120477.20

${ }^{1} \mathrm{~S} J$ Hepworth, 'L K Fraser, ${ }^{1,2}{ }^{1}$ E Petherick, ${ }^{3} \mathrm{~V} J$ Burley, ${ }^{3} \mathrm{~J}$ Cade, ${ }^{4} \mathrm{~T}$ de Kok, ${ }^{4} \mathrm{~S}$ van Breda, ${ }^{5} \mathrm{~S}$ A Kyrtopoulos, ${ }^{5} \mathrm{M}$ Botsivali, ${ }^{4} \mathrm{~J}$ Kleinjans, ${ }^{1} \mathrm{P}$ A McKinney. ${ }^{1}$ Paediatric Epidemiology Group, Division of Epidemiology, University of Leeds, Leeds, UK; ${ }^{2}$ Bradford Institute for Health Research, Bradford Royal Infirmary, Bradford, UK; ${ }^{3}$ Nutritional Epidemiology Group, Division of Epidemiology, University of Leeds, Leeds, UK; ${ }^{4}$ Department of Health Risk Analysis and Toxicology, University of Maastricht, Maastricht, The Netherlands; ${ }^{5}$ National Hellenic Research Foundation, Athens, Greece

Background Compounds created during the cooking and processing of food may be associated with to future child health if consumed during pregnancy. One such compound is acrylamide, which has been suggested as a potential dietary carcinogen, and is formed during the cooking of starchy foods. Little information is available on intake during pregnancy.

Objective To estimate intakes of the dietary compound acrylamide during pregnancy and describe the association of intakes with maternal age in years $(<20,20-39,40+)$, ethnicity and quintile of deprivation when classified using the index of multiple deprivation (IMD07).

Design Data were collected using a Food Frequency Questionnaire (FFQ) within the Born in Bradford multi-ethnic birth cohort study carried out in the city of Bradford, UK. The FFQ was given to all women recruited into Born in Bradford between September 2007 and July 2009 and completed at between 28-32 weeks of pregnancy. Participants were asked to self-complete information on the frequency of intakes for selected food items. Values for acrylamide intake were derived using estimated European exposure values taken from the literature within different food groupings. Values of acrylamide consumption were grouped into quintiles. Multivariate logistic regression was then used to investigate maternal characteristics associated with the highest quintile of acrylamide consumption.

Results 5294 women were recruited between September 2007 and July 2009 and 4212 (79.5\%) completed the FFO. Overall estimated mean intake of acrylamide was $35.5 \mu \mathrm{g} /$ day (SD: 27.2). French fries $(31.1 \%)$, toast $(18.3 \%)$ and crisps $(12.2 \%)$ made the largest contributions to overall intake levels. Maternal characteristics associated with the highest quintile of acrylamide intake ( $>52.4 \mu \mathrm{g} /$ day) was an age less than 20 years old (OR: 2.0, 95\% CI 1.6 to 2.5 ), those aged 40 and over were less likely to be in this quintile (OR: $0.3,95 \% \mathrm{CI}$ 0.1 to 0.7 ) when compared to pregnant women aged $20-39$ years. There was a significant interaction between ethnicity and deprivation levels $(p=0.01)$ with pregnant women of white ethnicity showing a increasing trend of being in the highest quintile of intake with increasing deprivation score whereas for women of south Asian ethnicity there was no significant relationship between deprivation and acrylamide intake.

Conclusion Intakes of the dietary contaminant acrylamide during pregnancy were found to vary according to maternal age, ethnicity and deprivation levels. This may have implications for the targeting of public health messages if there are future findings which lead to recommendations for reductions in intake.

\section{P21 INFLUENCE OF LIFE-COURSE SOCIO-ECONOMIC POSITION ON PAIN INTERFERENCE IN A PROSPECTIVE COHORT STUDY OF OLDER ADULTS}

doi:10.1136/jech.2010.120477.21

R J Lacey, J Belcher, P R Croft. Arthritis Research Campaign National Primary Care Centre, Keele University, Keele, Staffordshire, UK

Objective Pain that interferes with daily activities is the dominant cause of locomotor disability in older people. The objective of this study was to determine the influence of different trajectories of lifecourse socio-economic position (SEP) on pain interference in a population of older adults.

Design Prospective cohort study.

Setting General population in North Staffordshire, UK.

Participants All patients aged $50+$ registered with three different general practices were sent a baseline postal questionnaire. Responders consenting to further contact were followed up twice. Ethical approval was granted for all stages of the study.

Main Outcome Pain interference measured using an SF-36 item, asking how much pain interfered with normal work and housework during the last 4 weeks. Response categories dichotomised into 